\title{
Automated Detection of Extradural and Subdural Hematoma for Contrast-enhanced CT Images in Emergency Medical Care
}

\author{
Takeshi Hara*a, Naoto Matoba ${ }^{\mathrm{a}}$, Xiangrong Zhou ${ }^{\mathrm{a}}$, Shinya Yokoi ${ }^{\mathrm{a}}$, Hiroaki Aizawa ${ }^{\mathrm{a}}$ \\ Hiroshi Fujita ${ }^{a}$, Keiji Sakashita ${ }^{b}$, Tetsuya Matsuokab \\ ${ }^{a}$ Department of Intelligent Image Information, Graduate School of Medicine, Gifu University \\ 1-1 Yanagido, Gifu, Gifu 501-1194, Japan \\ ${ }^{\mathrm{b}}$ Department of Critical Medical Care, Senshu Critical Care Medical Center \\ 2-24 Rinku Ouraikita, Izumisano, 598-0048 Osaka, Japan
}

\begin{abstract}
We have been developing the CAD scheme for head and abdominal injuries for emergency medical care. In this work, we have developed an automated method to detect typical head injuries, rupture or strokes of brain. Extradural and subdural hematoma region were detected by comparing technique after the brain areas were registered using warping. We employ 5 normal and 15 stroke cases to estimate the performance after creating the brain model with 50 normal cases. Some of the hematoma regions were detected correctly in all of the stroke cases with no false positive findings on normal cases.
\end{abstract}

Keywords: contrast enhance CT scans, ER, CAD, brain, hematoma, head, automated detection

\section{INTRODUCTION}

The presence of specialists cannot be always expected in emergency room (ER). Head and abdominal injuries will be a cause of death in traffic accidents or disasters. Highly qualified staffs for emergency care will help casualties in fatal situations, but physicians in general hospitals may oversight important findings, which ER staffs should interpret. The ER staff often recognize hematoma region in brain using computed tomography (CT) images because of the limitation of treating time. A specific computer-aided detection (CAD) system will be of assistance to unaccustomed physicians to provide ER cares. We have been developing the CAD scheme for head and abdominal injuries. In this work, we have developed an automated method to detect hematoma regions in brain by comparing patients' scans with a CT brain model. Many researchers have been reporting brain models of MR and PET images to recognize the brain function using statistical parametric mapping (SPM) [1-4], however, there are no reports for statistical models for X-rays absorption of brain morphology using CT scans. In this work, we have assembled a CT brain model based on CT Hounsfield units (HU) to compare patients CT scans, and we apply the comparison technique to detect hematoma regions, typical morphological changes in ER images in brain, as an initial approach to utilize the brain CT model.

\section{METHODS}

\subsection{CT brain model}

Extraction approach for brain regions are illustrated in Fig. 1. Skull regions are extracted from original CT images (Fig.2 (a)) using an thresholding technique at $84 \mathrm{HU}$ slice by slice. Fig. 2(b) shows the extracted result of Fig. 2(a). The midlines in brain are determined by using the extracted bone boundaries. Fig. 2(c) shows the boundaries of the skull on a slice. To determine the midlines in brain, two points of frontal crest and internal occipital protuberance are recognized in each scan

*hara@info.gifu-u.ac.jp, takeshi.hara@mac.com; phone 81-58-230-6511; fax 81-58-230-6514

Medical Imaging 2007: Computer-Aided Diagnosis, edited by Maryellen L. Giger, Nico Karssemeijer, Proc. of SPIE Vol. 6514, 651432, (2007) · 1605-7422/07/\$18 · doi: 10.1117/12.710307 


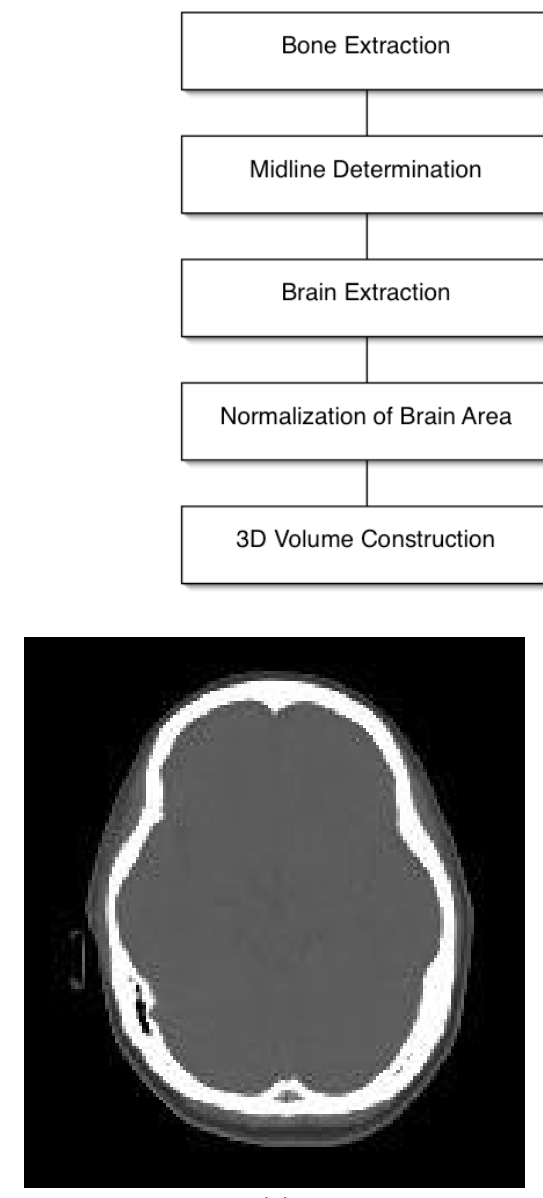

(a)

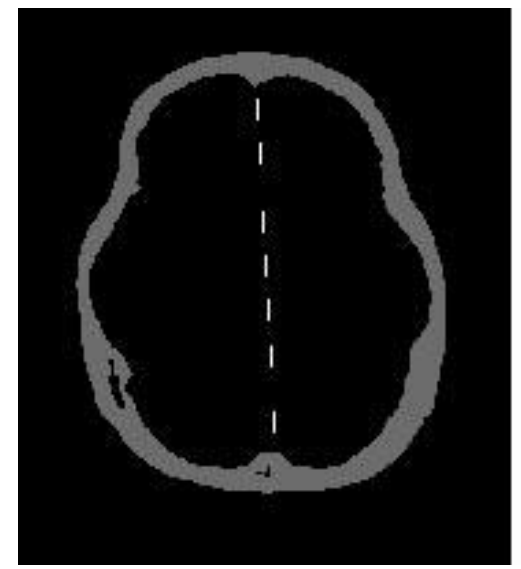

(d)

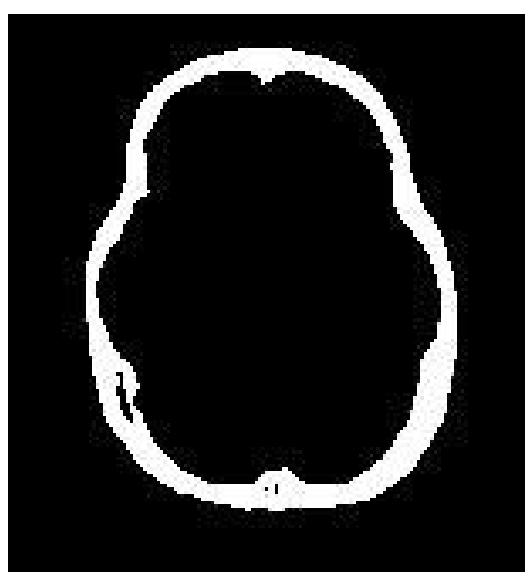

(b)

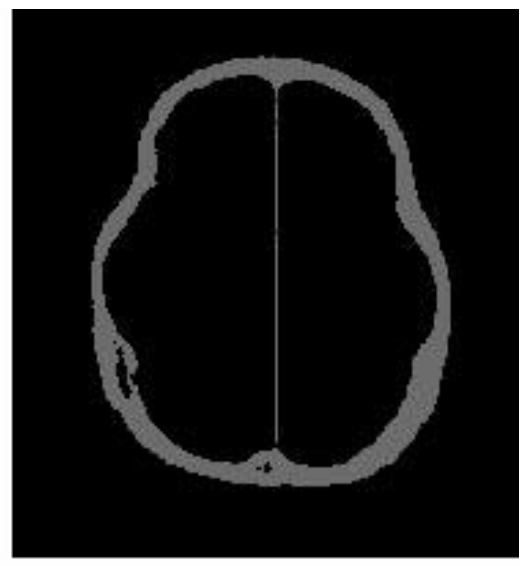

(e)

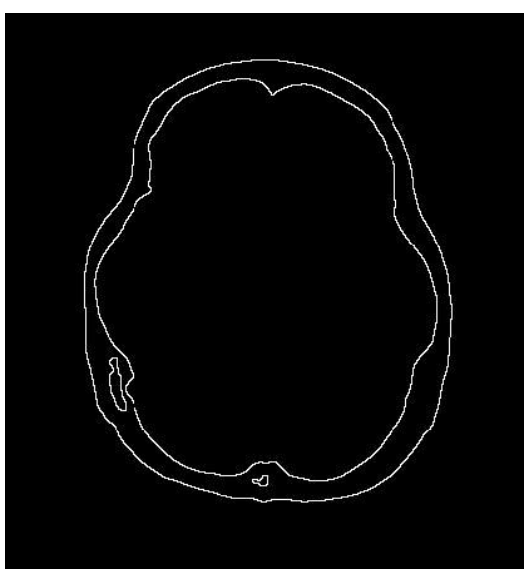

(c)

Fig. 2 Examples of CT images in brain in each processing.

(a) Original CT image.

(b) Bone (skull) extraction.

(c) Skull boundaries to determine the midline.

(d) Estimated midline.

(e) Results of tilt correction.

using the results of the shape analysis of skull boundaries. The straight line in Fig. 2(d) shows the determined midlines after the two characteristic points on the skull surface. These procedures are iterated from the parietal to a bottom slice including hypophysial fossa with pituitary gland. The bottom slice was employed to determine the standard plane every cases to construct the brain model. In the brain region in this scan, the brain area is normalized into $350 x 400$ pixels. The brain CT model from various cases is constructed to summarize these scans into one volume data. 
The brain CT model includes two features of mean and standard deviation values in a position of $(\mathrm{x}, \mathrm{y}, \mathrm{z})$. The mean (M) and the standard deviation (SD) are calculated at every position of (x, y, z), so that the $\mathrm{M}$ and the SD are defined as two functions of $\mathrm{M}(\mathrm{x}, \mathrm{y}, \mathrm{z})$ and $\mathrm{SD}(\mathrm{x}, \mathrm{y}, \mathrm{z})$. Fig. 3 shows the example of $\mathrm{M}(\mathrm{x}, \mathrm{y}, \mathrm{z})$ and $\mathrm{SD}(\mathrm{x}, \mathrm{y}, \mathrm{z})$. Fig. 3(a) shows a single scan of the $\mathrm{M}(\mathrm{x}, \mathrm{y}, \mathrm{z})$. Corpus callosum and lateral ventricle are illustrated in this scan. The highlighted area in fig. 3(b) indicates miss-registration region because of the boundary changes of each brain surface.

\subsection{Scoring patients' scans using brain model}

Patients' cases are also deformed using the same procedure to construct the brain CT model. The CT values, $\mathrm{P}(\mathrm{x}, \mathrm{y}, \mathrm{z})$ in the deformed images in $3 \mathrm{D}$ space are normalized using the $\mathrm{M}(\mathrm{x}, \mathrm{y}, \mathrm{z})$ and $\mathrm{SD}(\mathrm{x}, \mathrm{y}, \mathrm{z})$ in corresponding positions of $(\mathrm{x}, \mathrm{y}, \mathrm{z})$.

$$
\operatorname{Score}(\mathrm{x}, \mathrm{y}, \mathrm{z})=\{\mathrm{P}(\mathrm{x}, \mathrm{y}, \mathrm{z})-\mathrm{M}(\mathrm{x}, \mathrm{y}, \mathrm{z})\} / \mathrm{SD}(\mathrm{x}, \mathrm{y}, \mathrm{z})
$$

The equation (1) shows the normalized values $\operatorname{Score}(\mathrm{x}, \mathrm{y}, \mathrm{z})$ of the patient in the model space.

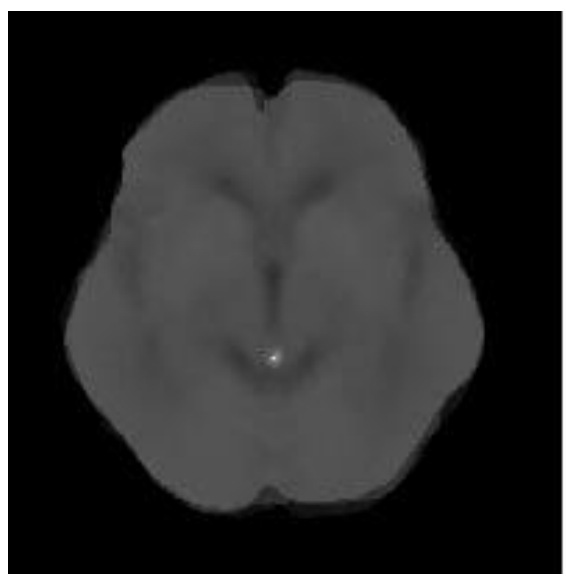

(a)

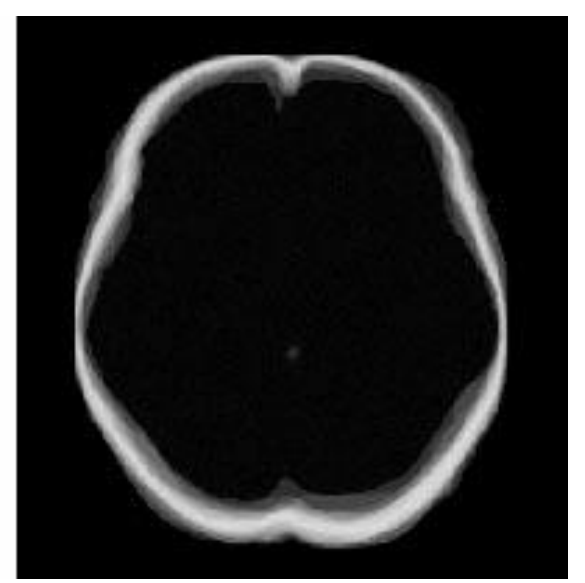

(b)

Fig. 3 Examples of mean and standard deviation images at a scan.

(a) Mean image. Image contrast is enhanced because of the printing quality.

(b) Standard deviation image.

\section{RESULTS}

Fifty-three normal and 15 abnormal CT cases were collected from a critical care medical center. Fifty cases out of 53 normal cases were employed to construct the brain CT model. A radiogolist in ER confirmed normal cases. Abnormal cases include subarachnoid hemorrhage (SAH), epidural hematoma, subdural hematoma, and cerebral contusion. Fourteen out of 15 abnormal cases have some irregular regions with high score of $\operatorname{Score}(\mathrm{x}, \mathrm{y}, \mathrm{z})$. One out of 15 abnormal case did not include those high score areas. Fig. 4 shows various example of Score $(x, y, z)$ images at a scan from 3D volume. Hematoma regions corresponding to each disease (Fig. 4(a)-(d)) were highlighted because of the measurement results of Score (x, y, z). On the other hand, the normal case in Fig. 4(e) shows a surface in Score(x, y, z) image, although the boundary has high scores because of the miss-registration in the brain CT model.

\section{CONCLUSIONS}

We employ 53 normal and 15 abnormal cases to estimate the performance after creating the brain CT model. The comparison patients' cases with the brain CT model indicates appropriate enhancement of hematoma regions. The CT brain model and the scoring approach may be useful to determine the degree of cerebral infarction in ER because the changing of CT value in brain has a relation to the blood flow.

\section{ACKNOWLEDGEMENTS}

This research is partly supported by Grand-in-Aid for Scientific Research from the Ministry of Education, Culture, 

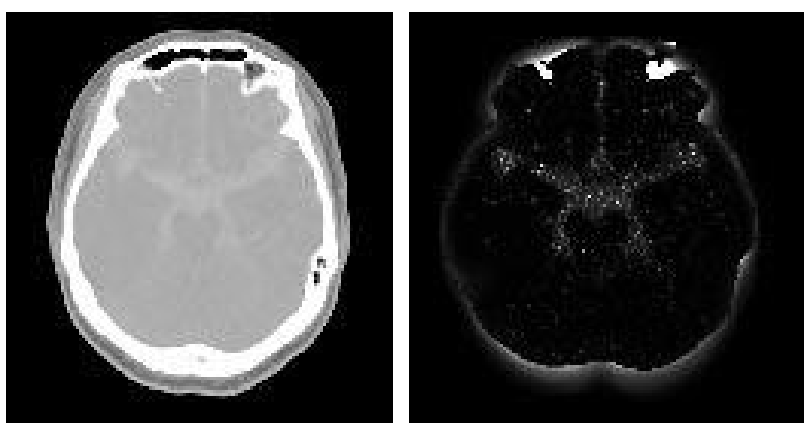

(a)
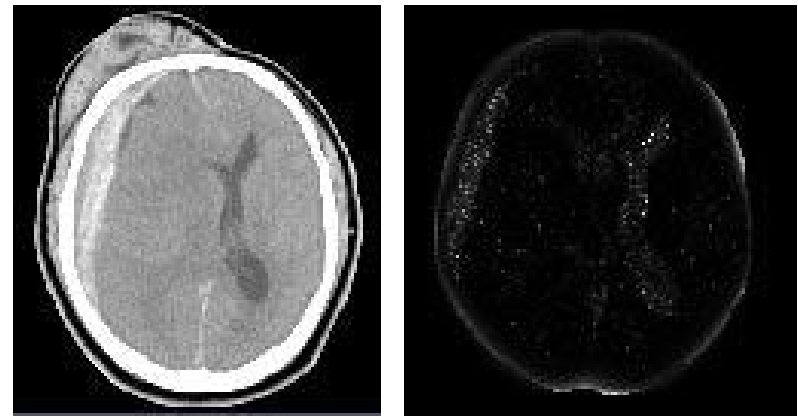

(b)
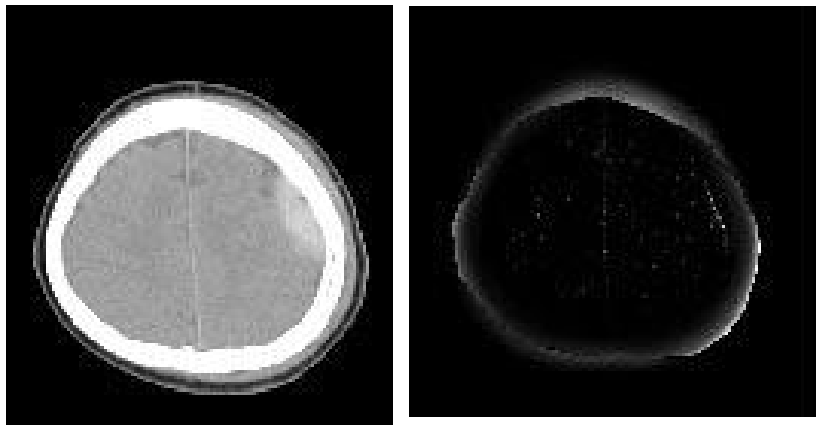

(c)
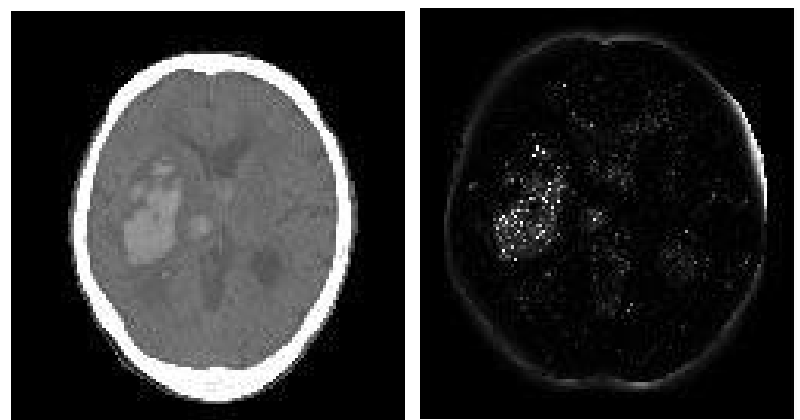

(d)
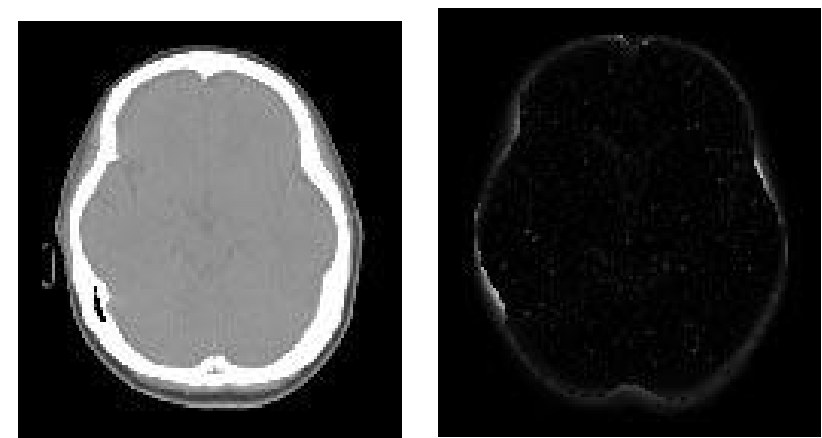

(e)

Fig. 4 Examples of original CT and Score images. Left image in each figure shows original CT scans from the volume data. Each right image indicate the corresponding Score with contrast enhancement for printing quarity.
(a) Subarachnoid hemorrage (SAH)
(b) Epidural hematoma
(c) Subdural hematoma
(d) Cerebral contusion
(e) Normal

Sports, Science, and Technology (MEXT), Japanese Government since 2003 and Daiwa Securities Health Foundation in 2005.

\section{REFERENCES}

1. McIntosh AR and Gonzalez-Lima F, "Structural equation modeling and its application to network analysis in functional brain imaging", Hum. Brain Mapp, 2, 2-22 (1994).

2. Friston KJ, Holmes A, Poline J-B, Price CJ, and Frith CD, "Detecting activations in PET and fMRI: levels of inference and power", NeuroImage, 4, 223-235 (1996).

3. Ashburner J, Neelin P, Collins DL, Evans A, and Friston K. " Incorporating priorknowledge into image registration", NeuroImage 6, 344-352 (1997).

4. Ashburner J, and Friston KJ, "Nonlinear spatial normalization using basis functions", Hum Brain Mapp, 7, 254-266 (1999).

5. Ashburner J, and Friston KJ, "Voxel-based morphometry--the methods", NeuroImage, 11, 805-821 (2000). 